Published in final edited form as:

Proc IEEE Int Symp Biomed Imaging. 2007 ; 4193392: 740-743. doi:10.1109/ISBI.2007.356958.

\title{
A CONSTRAINED NON-RIGID REGISTRATION ALGORITHM FOR APPLICATION IN PROSTATE RADIOTHERAPY
}

\author{
W. H. Greene ${ }^{1}$, S. Chelikani ${ }^{2}$, X. Papademetris ${ }^{1,2}$, J.P.S Knisely ${ }^{3}$, and J. Duncan ${ }^{1,2}$ \\ ${ }^{1}$ Department of Biomedical Engineering, Yale University, New Haven, CT, USA \\ 2Department of Diagnostic Radiology, Yale University, New Haven, CT, USA \\ ${ }^{3}$ Department of Therapeutic Radiology, Yale University, New Haven, CT, USA
}

\begin{abstract}
This paper presents a novel free-form deformation registration algorithm with non-rigid constraints to capture the transformation between the planning day and treatment day CT images used for external beam radiotherapy for prostate cancer. The algorithm is constrained to the predetermined motion of a segmented organ, which is described by an injective free-form deformation (FFD) based on Bsplines. The end goal is for the injective transformation to be used to update the radiotherapy plan to take into account bone and soft tissue deformation. The results of the algorithm have been compared to those achieved using rigid and fully non-rigid registration. The results clearly indicate that the constrained non-rigid registration algorithm presented in this paper performed much better at capturing the motion of the constrained organ, the bladder in this case, than the rigid or fully nonrigid registration algorithms.
\end{abstract}

\section{Keywords}

Image Registration; Biomedical Image Processing; Prostate Radiotherapy

\section{INTRODUCTION}

Prostate cancer is the most commonly diagnosed cancer among men in the United States. For the majority, external beam radiotherapy is a viable option, for the cancer is localized to the prostate in approximately $90 \%$ of new cases [1].

Recent advances in radiotherapy have led to three dimensional conformal radiotherapy (3DCRT) as well as intensity-modulated radiotherapy (IMRT). The aim of 3DCRT is to deliver a specified dose of radiation to the tumor, while minimizing normal tissue radiation exposure. In order to achieve maximum benefit, radiation beams from multiple directions are sculpted to conform to the shape of the tumor. With IMRT, the intensity of each radiation beam is modulated using three dimensional computed tomographic (3DCT) images to create an intensity pattern that best matches the shape of the tumor [2].

In both 3DCRT and IMRT, the radiation dose is delivered in multiple fractions over several weeks, thus correct patient positioning is necessary in order to ensure that the planned dosage of radiation is delivered to the prostate. Furthermore, recent efforts to deliver higher radiation dosages to the prostate while maintaining or lowering normal tissue exposure levels through IMRT require even more accurate positioning of the target volume and surrounding tissue at each treatment fraction [2]. 
At present, a planning day 3DCT image is acquired in order for the clinician to delineate the target volume and create a treatment plan. The treatment plan takes into account organ motion and patient positioning uncertainty in order to maximize dosage delivery to the tumor while minimizing normal tissue exposure levels. In addition, skeletal bone from digitally reconstructed radiographs (DRRs) created from the 3DCT data set are used to properly align the patient prior to therapy [2].

The most popular registration methods are dense field (intensity based) and sparse field (feature based) techniques, both of which can be used to register 3DCT data to two DRRs. Dense field methods are robust to noise, but are computationally expensive. Sparse field methods extract homologous features to estimate the proper patient position. Some methods extract natural features such as edges and ridges in the images, and others extract previously implanted fiducial markers. Sparse field algorithms are computationally inexpensive, but are limited by the accuracy of feature extraction [2].

Chelikani et al. extended the Minimax algorithm, which was initially a dense field metric, to use a mixture of dense and sparse field information. In addition, the method has the flexibility to use up to four portal images with full and reduced fields of view. The algorithm is now termed the Gradient Feature Weighted Minimax (GFW Minimax) method. The GFW Minimax was more accurate and robust in clinical settings due to the use of four portal images instead of two, which lowered the registration error. The downfall to this algorithm is that it does not account for organ motion, as it only utilizes rigid pelvic bony landmarks [2].

This paper presents a constrained non-rigid 3D-to-3D registration algorithm as a logical extension to the GFW Minimax method introduced by Chelikani et al. Accurate knowledge of important soft tissue structures (prostate, bladder, rectum) as well as bony structures (pelvis, left femur, right femur) will enable the clinician to set tighter planning margins around the target volume in the treatment plan. Escalated dosages can then be administered while maintaining or lowering normal tissue irradiation.

\section{METHODS}

The goal of this algorithm is to determine the injective transformation that non-rigidly maps the planning day image to the treatment day image, constrained to the predetermined transformation of an organ.

The algorithm consists of two steps. In the first step, a FFD model based on cubic B-splines is used to non-rigidly register the segmented organ from the planning day image to the same segmented organ from the treatment day image. In the second step, the FFD model is used to non-rigidly register the planning day image to the treatment day image, constrained to the known organ position.

\subsection{Hierarchical Multi-Resolution FFD Model}

Both steps of the algorithm employ a hierarchical multi-resolution FFD model to non-rigidly register the images [3]. The basic idea of FFDs is to deform an object by manipulating an underlying mesh of control points. The resulting control point configuration produces a smooth and $C^{2}$ continuous transformation. Choi et al. found that by restricting the range of the control points one can generate an injective FFD transformation [4]. The control point constraints discovered by Choi were used in the algorithm to produce an injective transformation.

To define a cubic B-spline FFD, we designate the domain of the image volume as $\Omega=\{(x, y$, $z) \mid 0 \leq x<X, 0 \leq y<Y, 0 \leq z<Z\}$, where $\Phi$ denotes a $(l+3) \times(m+3) \times(n+3)$ mesh of control points $\phi_{i, j, k}$ with uniform axial spacing $\delta_{x}, \delta_{y}, \delta_{z}$. The parameter domain of the image can now 
be defined as $\Theta=\{(u, v, w) \mid 0 \leq u \leq l, 0 \leq v \leq m, 0 \leq w \leq n\}$. The FFD can then be written as a $3 \mathrm{D}$ tensor product of $1 \mathrm{D}$ cubic B-splines:

$$
T_{0 d}(x, y, z)=\sum_{i=0}^{3} \sum_{j=0}^{3} \sum_{k=0}^{3} B_{i}(u-\lfloor u\rfloor) B_{j}(v-\lfloor v\rfloor) B_{k}(w-\lfloor w\rfloor) \phi_{(\lfloor u\rfloor+i)(\lfloor v\rfloor+j)(\lfloor w\rfloor+k)}
$$

where the relationship between the parameter and the image domain is straight foreword, $u=\frac{x}{\delta_{x}}, v=\frac{y}{\delta_{y}}, w=\frac{z}{\delta_{z}}$. In addition, $B_{i}$ represents the $i^{t h}$ basis function of the cubic B-spline:

$$
\begin{aligned}
& B_{0}(u)=\frac{(1-u)^{3}}{6} \\
& B_{1}(u)=\frac{\left(3 u^{3}-6 u^{2}+4\right)}{6} \\
& B_{2}(u)=\frac{\left(-3 u^{3}+3 u^{2}+3 u+1\right)}{6} \\
& B_{3}(u)=\frac{u^{3}}{6}
\end{aligned}
$$

B-splines are locally controlled, which makes them computationally efficient, even for a large number of control points. Local control means that cubic B-splines have limited support, such that changing one control point only affects the transformation in the local neighborhood of the manipulated control point.

Pseudovertices are used to ensure that the control volume being deformed by the control point mesh spans the entire image volume. The pseudovertices are the last control points along each axis and do not lie within the image domain. In order to guarantee that the control volume of the control point mesh, when deformed, spans the entire image volume, the last three control points, two contained within the image domain and one outside the image domain (pseudovertice), must not be manipulated.

The degree of non-rigid motion which can be captured depends on the resolution of the control points $\Phi$, which are the parameters of the cubic B-spline FFD. In order to create a computationally efficient algorithm with an adequate degree of non-rigid deformation required to capture the soft tissue motion with in the CT images, a hierarchical multi-resolution approach in which the resolution of the control point mesh is increased in a coarse to fine fashion was implemented [3].

Let $\Phi_{1}, \Phi_{2}, \cdots, \Phi_{N}$ denote a hierarchy of control point meshes, each increasing in resolution over the previous mesh. $\bar{T}_{1}, \bar{T}_{2}, \cdots, \bar{T}_{N}$ are the deformation functions associated with each control point mesh. The composition of all deformation functions, $\bar{T}=\bar{T}_{N} \circ \cdots \circ \bar{T}_{2} \circ \bar{T}_{1}$ is an injective mapping which defines the deformation of image volume $\Omega$. Given this, the final transformation is a composition of the previous injective transformations from each different control point resolution level and takes the following form:

$$
\bar{T}(\Omega)=\bar{T}_{N} \circ \cdots \circ \bar{T}_{2} \circ \bar{T}_{1}(\Omega)
$$

where $\bar{T}(\Omega)$ is the deformed image volume, which forms a voxel wise injective mapping to carry the planning day image into the treatment day image. 


\subsection{Injectivity Conditions}

Choi presented sufficient conditions for the local injectivity of a 3D uniform cubic B-spline function. An injective function is a function which has a unique output for each unique input. Given

$$
\begin{aligned}
& T: U \rightarrow V \\
& S: V \rightarrow U
\end{aligned}
$$

then T is injective if $S(T(u))=u$ for every $u$ in $U$. The injective conditions presented are used in this algorithm to ensure the injectivity of the transformation [4].

The injective constraints simply constrain each control point to move within a local sphere of radius $r<R$, where $R \approx 0.4033$ of the control point spacing. To clarify, let $\Delta \phi_{i j k}$ be the

displscement of control point $\phi_{i j k}$ from $\phi_{i j k}^{0}$, that is $\Delta \phi_{i j k}=\phi_{i j k}-\phi_{i j k}^{0}=\left(\Delta x_{i j k}, \Delta y_{i j k}, \Delta z_{i j k}\right)$. Let $\delta_{x}=\max \left\{\left|\Delta x_{i j k}\right|\right\}, \delta_{y}=\max \left\{\left|\Delta y_{i j k}\right|\right\}, \delta_{z}=\max \left\{\left|\Delta z_{i j k}\right|\right\}$. With this, the function $T_{0 d}$ is locally injective all over the domain if $\delta_{x}<R, \delta_{y},<R, \delta_{z}<R$ [4].

\subsection{Segmented Organ Registration}

The first step of the algorithm employs a FFD model, as described above, to non-rigidly register the segmented organ from the planning day image to the segmented organ from the treatment day image. The injective constraints as laid out by Choi are used to ensure the transformation is smooth and does not locally fold [4].

At the end of each step in the hierarchical multi-resolution registration, the deformed control point mesh and associated transformed image, $\left[I_{1}, \Phi_{1}\right],\left[I_{2}, \Phi_{2}\right], \cdots,\left[I_{N}, \Phi_{N}\right]$, are saved to file to be later used as constraints.

\subsection{Constrained Image Registration}

The second step of the algorithm performs a constrained non-rigid registration in order to determine the transformation that maps the planning day image into the treatment day image. The transformation is constrained to the transformation of the soft tissue object which is determined in the first step.

The same initial control point meshes that are used to register the segmented organ are used in the second step, enabling the saved deformed control point meshes from the prior step to be used as constraints. Each registration level $n$ has an associated deformed control point grid $\Phi^{n}$ and deformed image $I^{n}$. Control points $\phi_{i j k}^{n}$ located within the segmented organ in image $I^{n}$ are used as constraints for control points $\phi_{i j k}$ in the current registration by setting $\Delta \phi_{i j k}=\Delta \phi_{i j k}^{n}$.

\section{RESULTS}

The constrained non-rigid registration algorithm has been tested on real clinical data from patients undergoing external beam prostate radiotherapy. The original CT images had a size of $512 \times 260 \times 512$ voxels and spatial resolution of $1.17 \mathrm{~mm} \times 1.25 \mathrm{~mm} \times 1.17 \mathrm{~mm}$, but were resliced to a size of $82 \times 56 \times 42$ voxels and spatial resolution of $3.94 \mathrm{~mm} \times 4.19 \mathrm{~mm} \times$ $3.91 \mathrm{~mm}$ to decrease the run time of the algorithm.

For comparison, the planning day and treatment day image were registered using a fully nonrigid registration algorithm, as well as a rigid registration algorithm. 
Visually, both algorithms performed poorer than the constrained non-rigid registration algorithm. The rigid registration algorithm performed exceptionally poor, for it estimated the transformation to be the identity transform. In order to analytically assess the quality of the registration in the clinical data, the percentage of bladder and prostate volume overlap was calculated for all three different registration algorithms. In addition, the overlap was also calculated without registration. As expected, the constrained non-rigid registration algorithm performed significantly better in aligning the bladder than did the fully non-rigid or rigid registration. Because the hard bladder constraints acted as soft constraints for local tissue, pulling local tissue along, the algorithm presented in this paper better aligned the prostate than either the rigid or fully non-rigid registration. Table 1 summarizes the results of the registration.

\section{CONCLUSION AND FUTURE WORK}

A constrained non-rigid registration algorithm has been developed. The free-form deformation (FFD) model is used in a hierarchical multi-resolution approach in order to produce a computationally efficient algorithm with an adequate degree of non-rigid deformation. This algorithm performed significantly better at aligning the bladder, which is an important issue in external beam radiotherapy, because exposing the bladder to high levels of radiation increases complications such as urinary incontinence.

The algorithm presented in this paper is still in its infancy. Eventually, the algorithm will be expanded to use the prostate, rectum, and bladder as non-rigid constraints, and the pelvis, right femur and left femur as rigid constraints. By forcing local bones and organs subject to radiation, due to their proximity to the prostate, to get mapped to their known position, the other soft tissue in the surrounding areas will be easily carried along in the final step of the algorithm.

An algorithm which can create an accurate injective mapping from the planning day CT to the treatment day CT, taking into account soft tissue deformation, will allow the clinician to quickly update the radiotherapy plan to the position of the organs on the treatment day. An updated radiotherapy plan which takes soft tissue into account will allow the clinician to tighten the planning margins around the target volume in the treatment plan. The clinician will also be able to increase radiation dosage while maintaining or lowering normal tissue irradiation, ultimately increasing the effectiveness of radiotherapy while reducing side effects.

\section{REFERENCES}

[1]. Potosky AL, Legler J, Albertsen PC, Stanford JL, Gilliland FD, Hamilton AS, Eley JW, Stephenson RA, Harlan LC. Health outcomes after prostatectomy or radiotherapy for prostate cancer: Results from the prostate cancer outcomes study. J. Nat. Cancer Inst October;2000 92:1582-1592. [PubMed: 11018094]

[2]. Chelikani S, Purushothaman K, Knisely J, Chen Z, Nath R, Bansal R, Duncan J. A gradient feature weighted minimax algorithm for registration of multiple portal images to 3DCT volumes in prostate radiotherapy. Int. J. Radiation Oncology Biol. Phys 2006;65:535-547.

[3]. Rueckert D, Sonoda L, Denton E, Rankin S, Hayes C, Leach MO, Hill D, Hawkes DJ. Comparison and evaluation of rigid and non-rigid registration of breast MR images. SPIE 1999;3661:78-88.

[4]. Choi, Y.; Lee, S. Local injectivity conditions of 2D and 3D uniform cubic b-spline functions. 1999. p. $302-311$. 


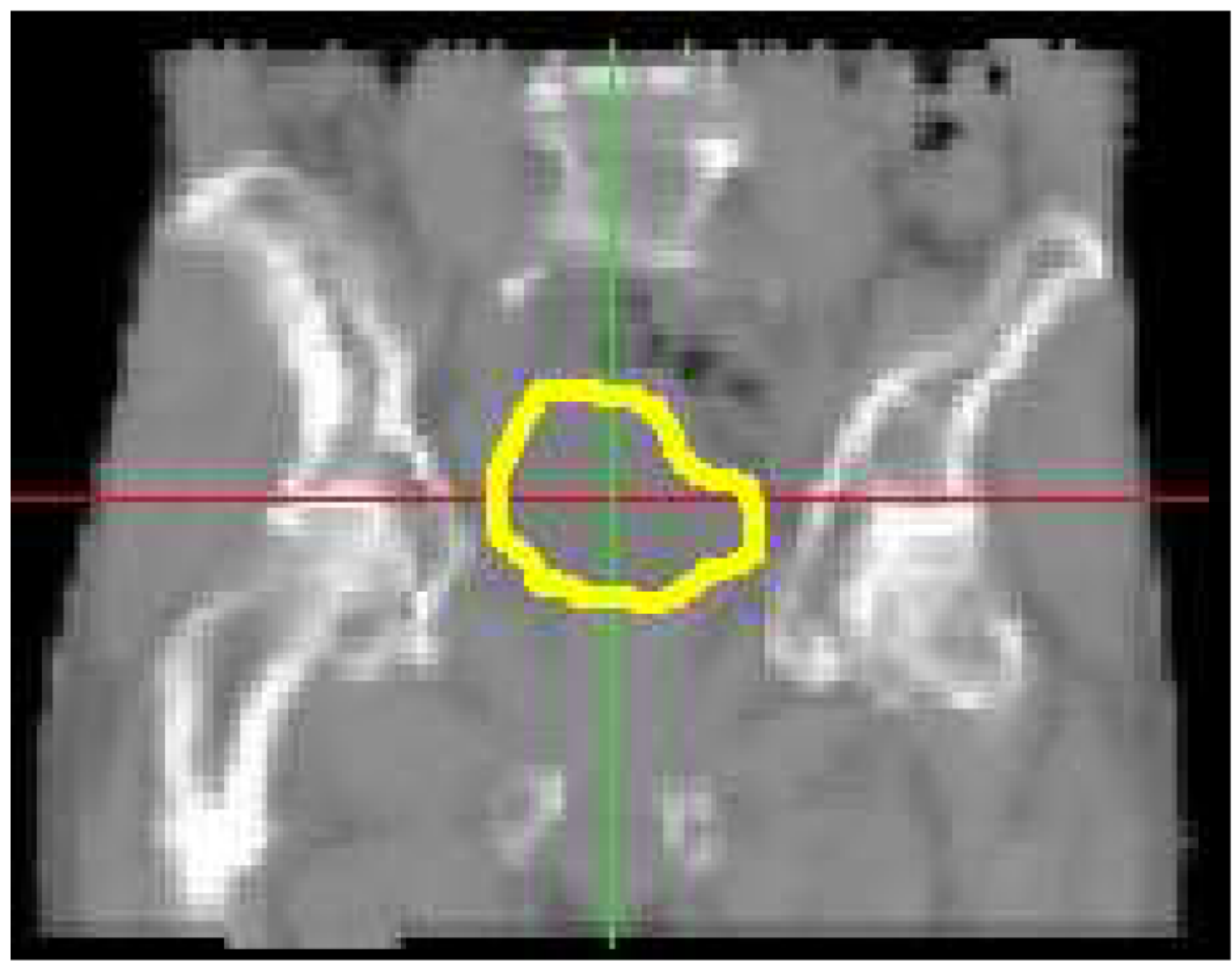

Fig. 1.

Bladder outline from constrained non-rigid registration. 


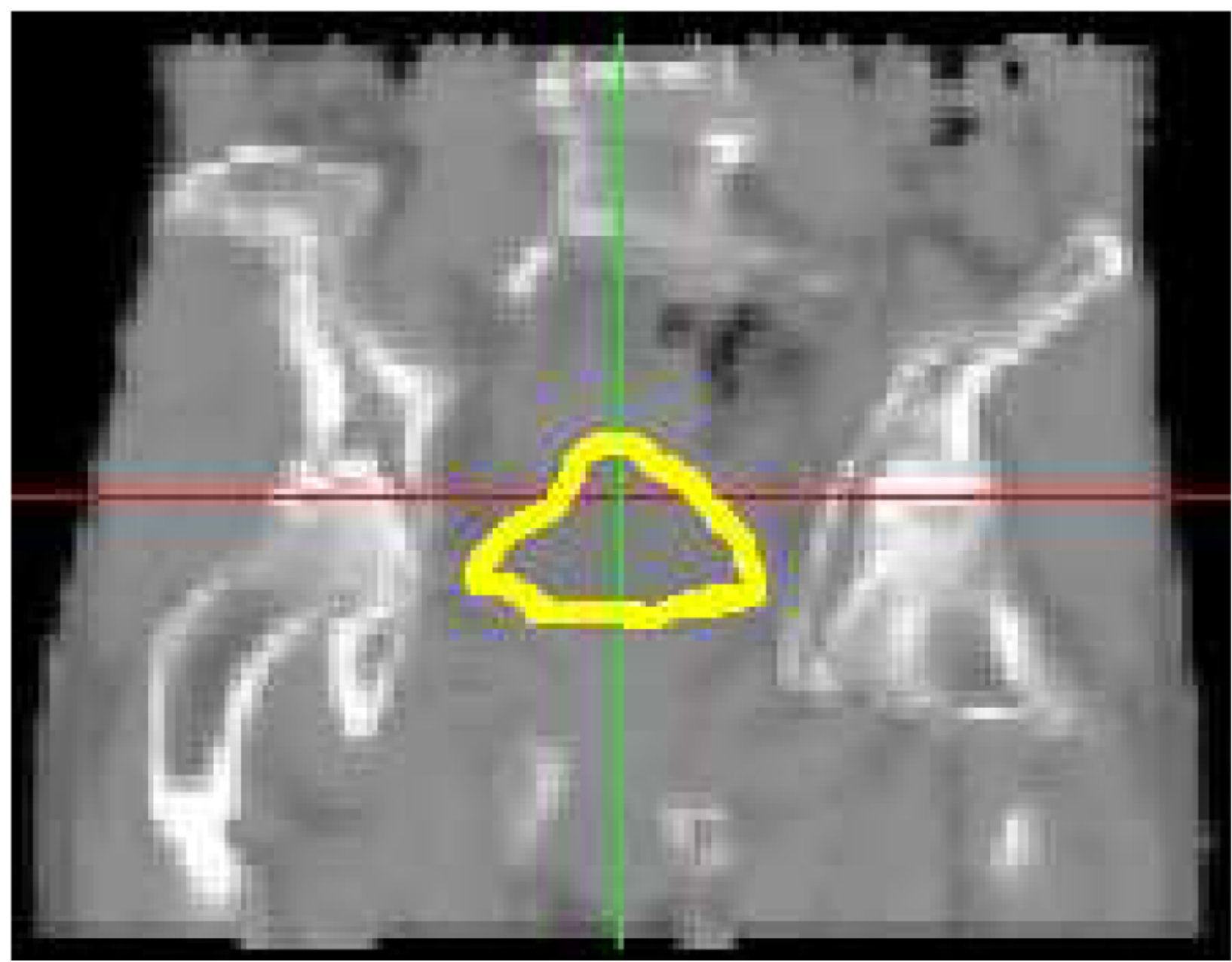

Fig. 2.

Bladder outline from fully non-rigid registration. 


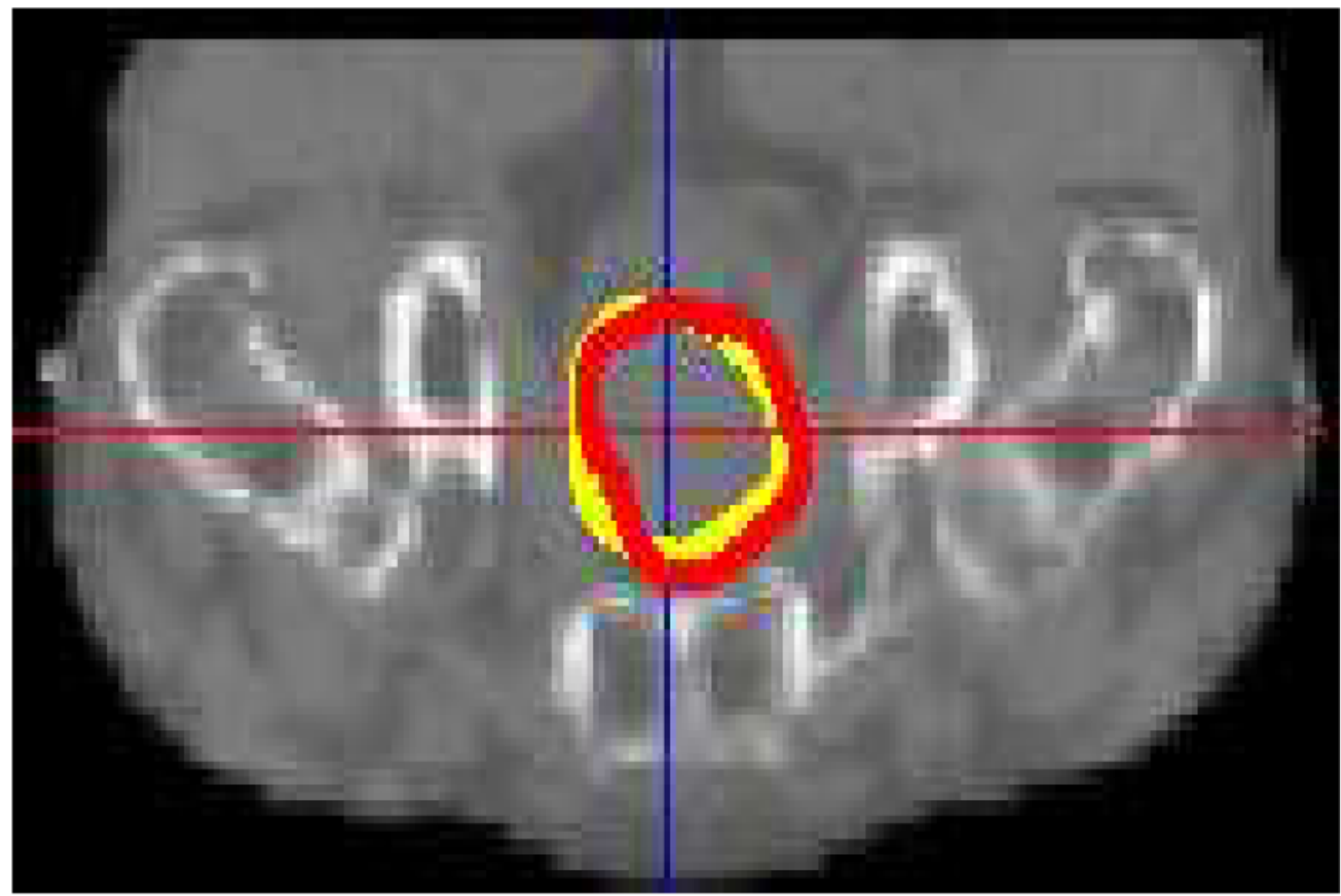

Fig. 3.

Prostate outline from constrained non-rigid registration overlaid on treatment day image. 


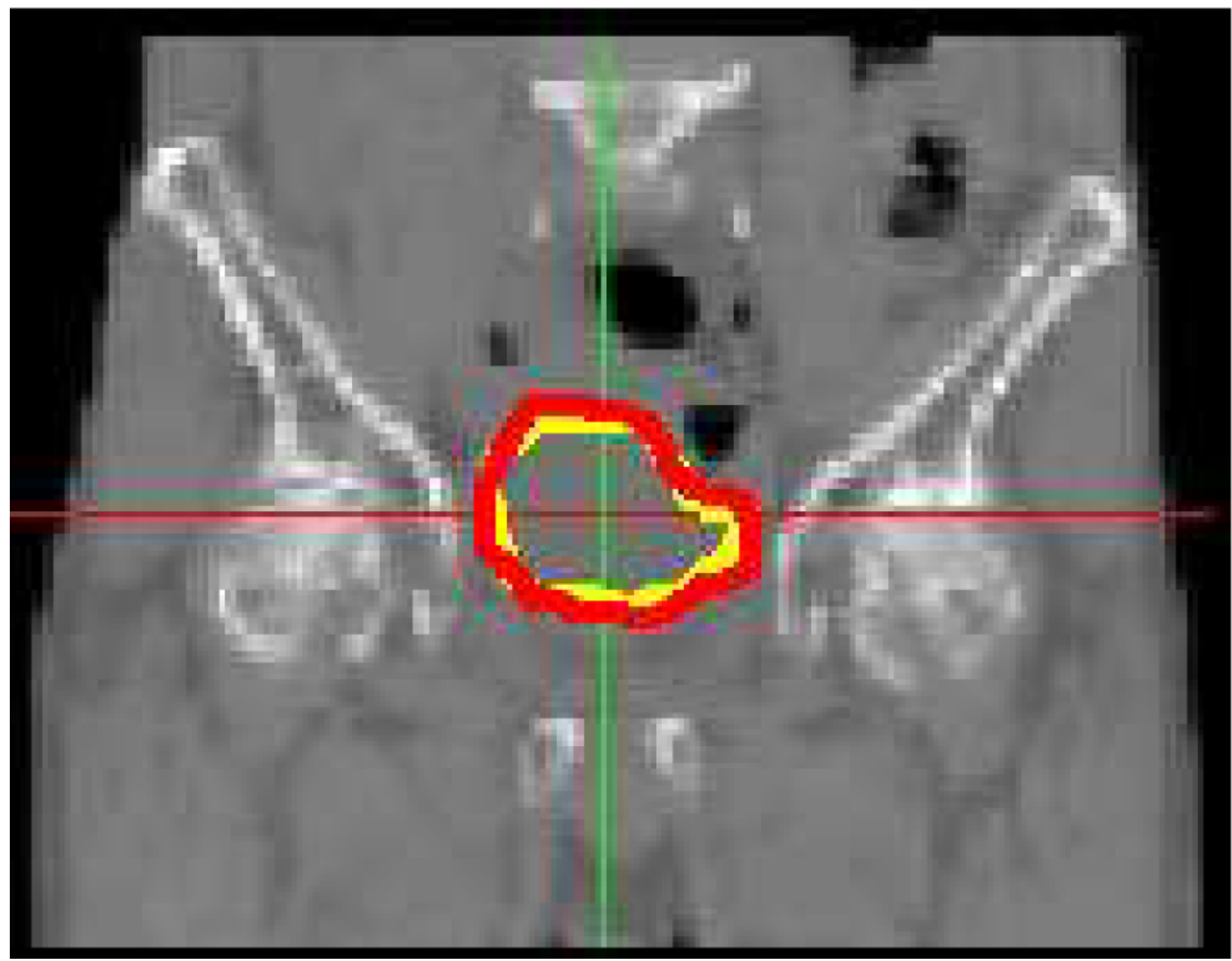

Fig. 4.

Bladder outline from constrained non-rigid registration overlaid on treatment day image. 
Table 1

Registration Results

\begin{tabular}{|c|c|c|}
\hline Registration & Bladder Volume Overlap & Prostate Volume Overlap \\
\hline Constrained (B1) Non-Rigid & $86.42 \%$ & $74.63 \%$ \\
\hline Non-Rigid & $75.17 \%$ & $61.95 \%$ \\
\hline Rigid & $65.44 \%$ & $47.06 \%$ \\
\hline None & $65.44 \%$ & $47.06 \%$ \\
\hline
\end{tabular}

